\title{
Localization of Weak Heat Sources in Electronic Devices Using Highly Sensitive Lock-in Thermography
}

\author{
J. P. Rakotoniaina, O. Breitenstein, and M. Langenkamp \\ Max-Planck-Institut für Mikrostrukturphysik, Weinberg 2, D-06120 Halle, Germany \\ Phone :+49-345-5582-760, Fax +49-345-5511-223, E-mail: pati@mpi-halle.de
}

\begin{abstract}
Using lock-in thermography the temperature resolution of a Focal Plane Array (FPA) thermocamera can be improved down to $40 \mu \mathrm{K}$ after $1000 \mathrm{~s}$ measuring time. This allows the detection of even weak heat sources (hot spots) in electronic devices. The technical realization of lock-in thermography is described here with typical applications to the investigations of shunts in solar cells and localization of local heat sources in ICs. Because of its high spatial resolution, its high thermal sensivity as well as its simplicity, this technique is an advantageous alternative to usual thermal testing in electronic devices.
\end{abstract}

Keywords: solar cells, IC testing, thermography

\section{Introduction}

Defects in electronic devices like leakage currents or short circuits in integrated circuits (Ics) or solar cells are sources of local heat in such devices. For devices having a poor heat conductivity as e.g. thin film solar cells on glass substrate it is possible to localize these heat sources by stationary thermography with a good spatial resolution of $0.1 \mathrm{~mm}$ [1]. Silicon substrates, however, have a much higher heat conductivity so that locally dissipated heat rapidly spreads both laterally and into the depth of the sample. This leads to a "blurred" image and a lower temperature contrast, which in many cases cannot be detected anymore by stationary thermography.

Hot spots in electronic devices are usually imaged using nematic or thermochrome liquid crystals, giving a thermal resolution in the range of $100 \mathrm{mK}$ [2]. Using Fluorescence Microthermal Imaging (FMI), which is based on the strong temperature dependence of certain fluorescent dyes covering the surface, a temperature resolution up to $10 \mathrm{mK}$ can be obtained [3]. However, even this temperature resolution may not be sufficient to detect power sources in the range below one $\mathrm{mW}$. Furthermore, due to the high lateral heat conductivity in silicon, the heat sources appear significantly broadened in stationary thermography, and the use of a covering on the surface is in some cases not tolerable.

Using lock-in techniques the detection limit of IR thermography can be drastically improved below $100 \mu \mathrm{K}$ owing to the signal averaging. Here the spatial resolution is related to the thermal diffusion length, which decreases with the square root of the lockin frequency and has a value of about $1 \mathrm{~mm}$ at $\mathrm{f}_{\text {lock-in }}=30 \mathrm{~Hz}$ for silicon. For microscopic surface-near heat sources, however, the spatial resolution may be well below the thermal diffusion length as will be shown below. Using a microscope objective the lateral resolution of lock-in thermography may be as good as $5 \mu \mathrm{m}$.

In this paper we describe the principle of lock-in thermography. Then applications to the investigation of shunts in solar cells and the detection of weak heat sources in 
integrated circuits (ICs) are introduced to demonstrate the potential of the lock-in thermography technique. For solar cell investigations lock-in thermography enables the non-destructive localization and the quantitative analysis of internal shunts. Local heat sources in ICs may be caused by heat dissipation in the normal operation of the IC, by electrostatic pulse (ESP)-induced leakage sites, by shorts between metallization lines, or by other kinds of internal faults. After a defect has been localized by lock-in thermography, microscopic analytical techniques (e.g. TEM with focused ion beam preparation or SEM techniques) have to be used to understand the physical reason for this defect.

As will be shown below, lock-in thermography doesn't need any surface preparation, it is not sensitive to stray light or temperature drifts, and it shows a unique thermal sensivity. Therefore lock-in thermography is an advantageous alternative to liquid crystal and fluorescence microthermal investigations (FMI), which are presently widely used in thermal electronic device testing.

\section{Principle of the Lock-in Thermography and its technical realization}

The lock-in thermography technique has already become an established technique of the nondestructive testing of materials and devices [4]. Its principle consists in introducing periodically modulated heat into an object and monitoring only the periodic surface temperature modulation, phase referred to the modulated heat supply. For electronic devices the heat modulation most simply occurs by applying a pulsed bias. The surface temperature is measured with an IR (infrared) thermocamera and the information of each pixel of the incoming images is processed as it were fed into a lock-in amplifier.

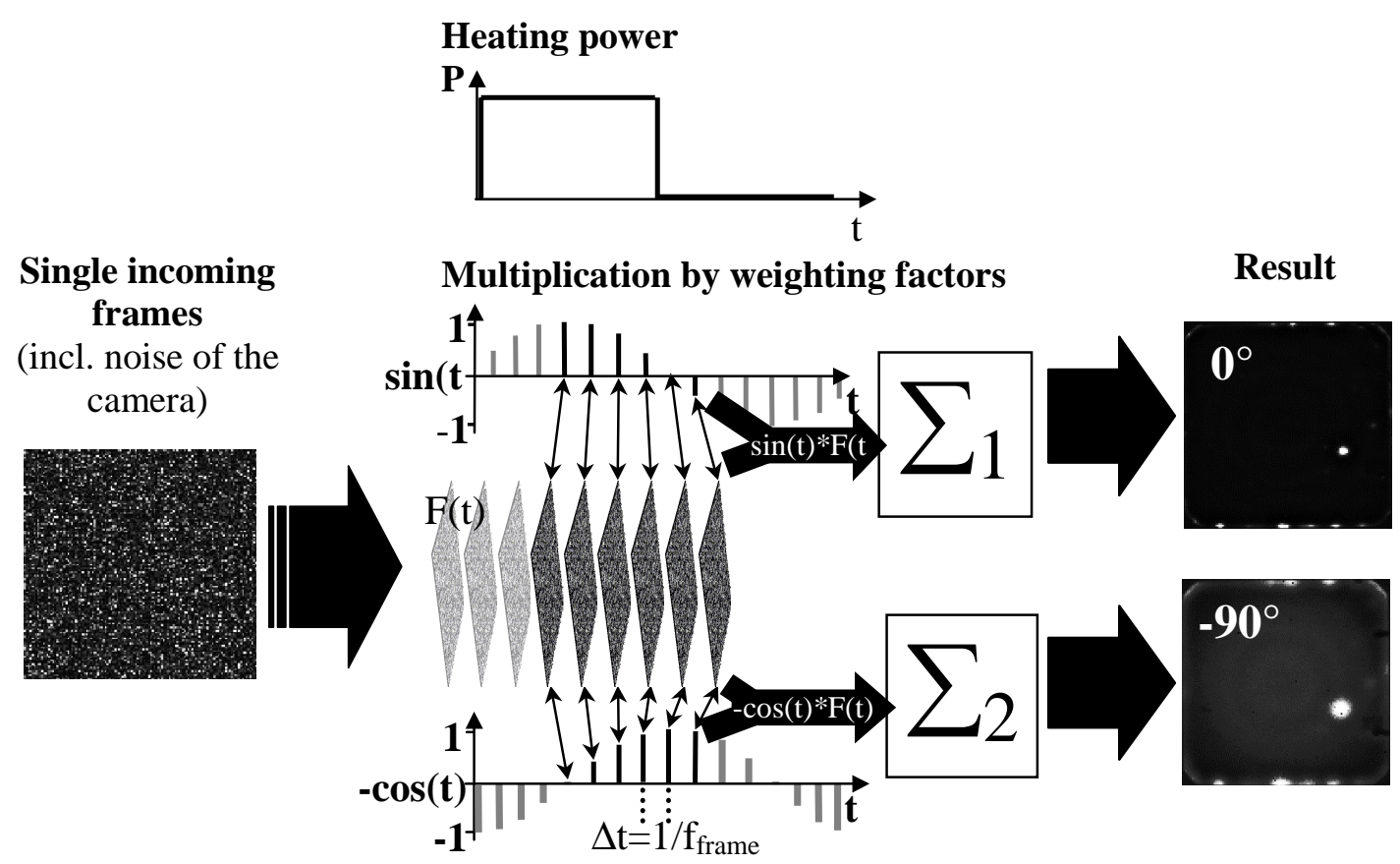

Fig. 1: The principle of lock-in thermography, here shown for $\mathrm{f}_{\text {lock-in }}=\mathrm{f}_{\text {frame }} / 16$

The digital lock-in correlation procedure (Fig.1) consists in successively multiplying the incoming IR images by a set of weighting factors and summing up the results in a frame grabber. The weighting factors are an approximation of a harmonic function and are 
synchronized to the pulsed bias applied to the sample. The number of frames per lock-in period governs the lock-in frequency. Since both the amplitude and the phase of the measured surface temperature modulation may change with the position, a 2-phase lockin detection has to be used. Thus, a lock-in measurement can produce either an amplitude- and phase-image, or an in-phase $\left(0^{\circ}\right)$ and in quadrature $\left(-90^{\circ}\right)$ image, both referring to the phase of the heat supply. For the detection of local heat source in electronic devices the amplitude signal is the most informative one, because it is directly proportional to the locally dissipated power.

Dynamic Precision Contact Thermography (DPCT [5]) was the first lock-in thermography technique which allowed to detect temperature modulations below 100 $\mu \mathrm{K}$. However, this technique worked in contacting mode and showed a spatial resolution of about $30 \mu \mathrm{m}$, hence it was not applicable to investigate integrated circuits. Then a lock-in thermography system based on a highly sensitive IR camera with a high frame rate was developed at MPI Halle. This system with $128 * 128$ pixel resolution shows a noise level of about $20 \mu \mathrm{K}$ after 1000s measure time and, combined with a special microscope objective, it can reach a spatial resolution of $5 \mu \mathrm{m}$. This was the first system which demonstrated the power of the microscopic lock-in thermography for investigating integrated circuits [6]. Based on this system Thermosensorik GmbH Erlangen [7] has developed the commercial system TDL 384 M 'Lock-in'. With a resolution of $384 * 288$ pixel and a noise level of $40 \mu \mathrm{K}$ after $1000 \mathrm{sec}$ measure time this system is the highest sensitive and highest resolution lock-in thermography system commercially available. The scheme of this system is presented in Fig. 2.

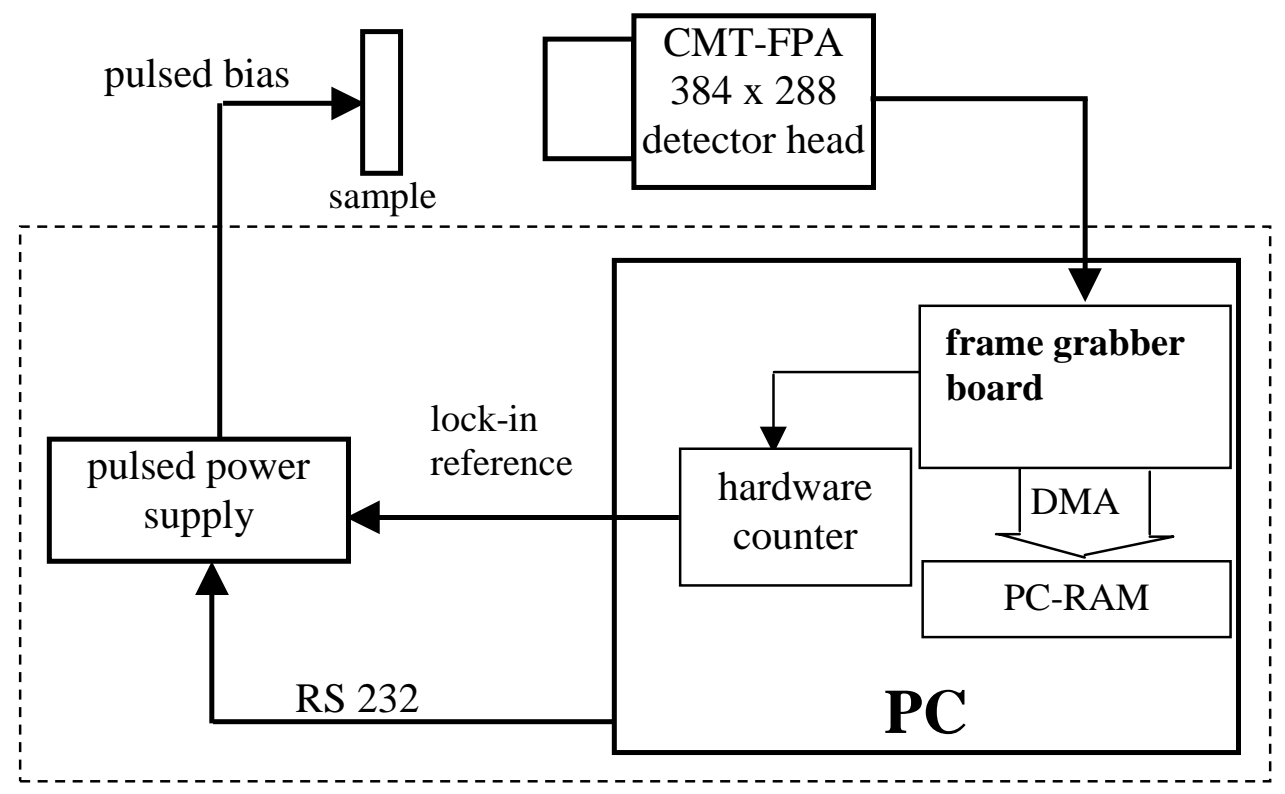

Fig.2: Scheme of the TDL 384 M 'Lock-in' system

The IR Detector head is based on a Stirling-cooled mercury cadmium telluride (MCT) midwave $(3-5 \mu \mathrm{m})$ Focal Plane Array. This array has a single detector size of $20 * 20 \mu \mathrm{m}^{2}$ and can be equipped with different IR objectives. Combined with a special microscope objective a spatial resolution of $10 \mu \mathrm{m}$ can be obtained, which may be lowered down to $5 \mu \mathrm{m}$ using a lens extender ring. Each pixel is digitized with a resolution of $14 \mathrm{Bit}$. In full frame mode the maximum possible frame rate is $140 \mathrm{~Hz}$, 
corresponding to a pixel transfer rate of $15.5 \mathrm{Mhz}$. The computer, which is a $2 * 800 \mathrm{Mhz}$ dual Pentium III running under Windows NT, allows to process all incoming data according to Fig. 1 online, hence no primary data have to be stored. The images are captured by a Matrix Vision frame grabber board, which writes the incoming frames directly into the RAM by direct memory access (DMA). The PC software picks up the frames out of the RAM for correlation. A programmable hardware counter is provided to ensure that only lock-in periods with a complete number of periods are used for correlation. The power supply for providing the sample bias is equipped with a solid state relay for pulsing the bias. For investigating small objects like ICs the system may be equipped with a stable vertical support and a $x-y-z$ movable device testing stage The noise level of this system is down to $44 \mu \mathrm{K}$ (effective value)after 1000s acquisition time and further reduces with $1 /(\mathrm{t})^{1 / 2}$.

\section{Applications}

All applications presented here are measured using the TDL $384 \mathrm{M}$ 'Lock-in' system with the resolution $288 * 288$ pixel in sub-frame mode, since these objects are essentially quadratic. Lock-in thermography has already been proven to be an established and efficient tool for the characterization of shunts in solar cells $[8,9]$. Shunts are local sites of an enhanced forward current. Therefore they reduce the open circuit voltage and the fill factor of solar cells. Fig. 3 shows lock-in thermography results of a $10 * 10 \mathrm{~cm}^{2}$ multicrystalline silicon cell measured with $0.5 \mathrm{~V}$ forward bias pulsed at the frequency of $4 \mathrm{~Hz}$. The measurement time for one lock-in image was $32 \mathrm{~min}$.
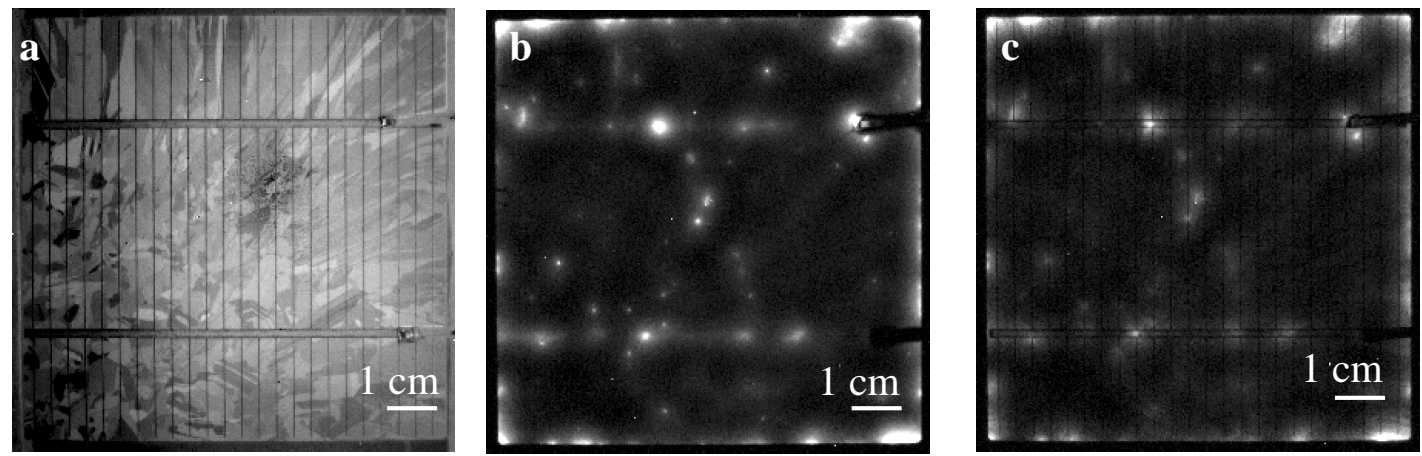

Fig.3: Topography image (a) of a solar cell and lock-in thermogram using an attached IR emitter foil (b) and without surface covering (c).

Fig. 3a shows the topography image of the solar cell as delivered directly by the camera. One can see the grid lines and the different grains owing to their different reflectivities. Figs. $3 \mathrm{~b}$ and $3 \mathrm{c}$ are lock-in thermograms (amplitude images) of this sample, both scaled from 0 to $1 \mathrm{mK}$ (effective value). In Fig. 3b the sample was covered by a vacuumattached $20 \mu \mathrm{m}$ thin black-painted plastic foil to achieve a high and homogenous IR emissivity [8], whereas in Fig. 3c the sample was not covered with this IR emitter foil. Using the IR emitter foil guarantees that the IR signal is independent on the IR emissivity of the sample, which is especially low at the metallic grid lines. However, the comparison shows that qualitatively both pictures contain the same information. This proves that solar cells may be investigated by IR lock-in thermography also without any IR-active surface covering, which makes this technique also interesting for in-line process control. In this solar cell we see that the dominant shunts are essentially point- 
like and are lying both at the edges and within the wafer. The edges shunts are caused by an insufficient "opening" of the pn-junction, which was done here by mechanical treatment of the cell edge. Some of the shunts in the area are lying below a grid line and seem to be caused by the emitter metallization. Owing to the low IR emissivity of the grid lines, especially these shunts appear weaker in the measurement without using the IR emitter foil (Fig. 3c). To analyze the nature of the shunts other methods have to be used as e.g. SEM (scanning electron microscopy), TEM (transmission electron microscopy), EBIC (electron beam induced current) or LBIC (light beam induced current).
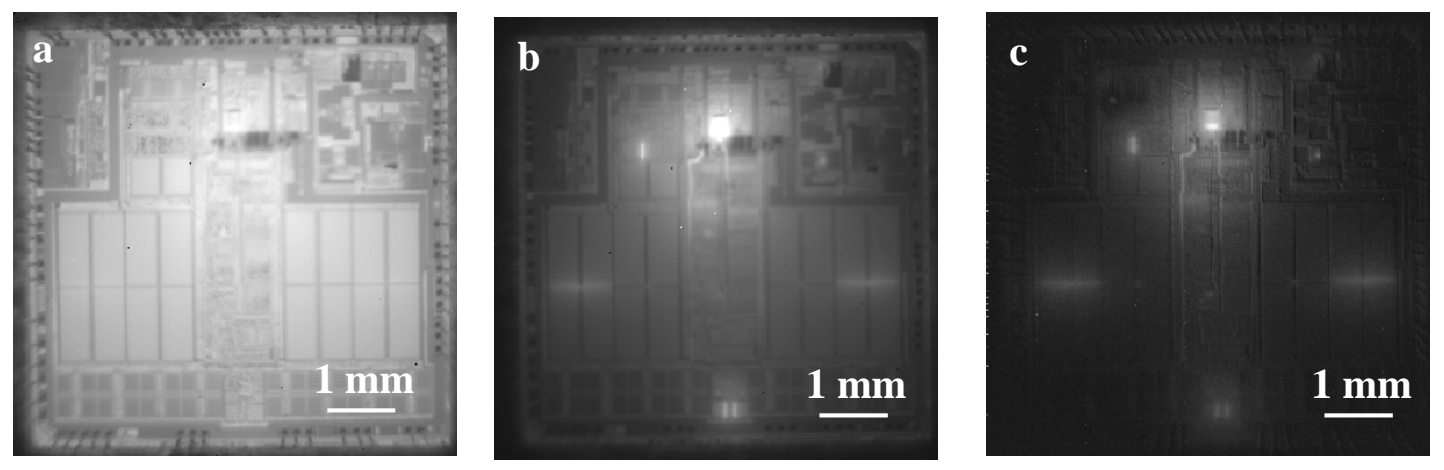

Fig 4. Lock-in thermogram of an IC measured at $0.1 \mathrm{~Hz}$ (a), at $4 \mathrm{~Hz}$ (b) and $36 \mathrm{~Hz}$ (c)

Another useful application of lock-in thermography is the electronic device testing of ICs with no additional surface preparation. Fig. 4 shows a series of lock-in thermograms (amplitude images) of an IC with an applied pulsed bias of $3 \mathrm{~V}$ measured at different lock-in frequencies. Since metallizations show a high reflectivity and a low IR emissivity, they generally appear dark also in the lock-in thermograms, thereby allowing an easy orientation on the surface of the IC. The aim of the use of different lock-in frequencies is to demonstrate the frequency dependence of the obtained images. The spatial resolution of lock-in thermography depends both on the heat source geometry and on the thermal diffusion length, which basically governs the extension of the "halo" around local heat sources coming from the heat conductivity of the material. The thermal diffusion length of silicon is about $1 \mathrm{~mm}$ at $30 \mathrm{~Hz}$ and decreases with the inverse of the square root of the lock-in frequency [10], hence a higher frequency leads to a better spatial resolution. On the other hand, with increasing lock-in frequency the magnitude of the temperature modulation decreases, hence the images become more and more noisy. Fig. 4a shows that at a frequency of $0.1 \mathrm{~Hz}$ (which is equivalent to stationary imaging) nearly the whole IC is heated up, and the contrast of the dominant heat source in the upper part of the IC is considerably broadened. With a frequency of $4 \mathrm{~Hz}$ except of the dominant heat source some other ones become clearly visible. At the frequency of $36 \mathrm{~Hz}$ (Fig. 4c) the heat sources appear even sharper due to the shorter thermal diffusion length and there are also more details visible. Note the different appearance of the weak heat source right from the mean hot spot in the different images. 


\section{Conclusions}

It has been shown that lock-in thermography is a nondestructive and highly sensitive method to localize weak heat sources in electronic devices. It has been used successfully for detecting local shunts in solar cells and for localizing weak heat sources in ICs. In comparison to previous microthermal imaging techniques like liquid crystal or fluorescence microthermal imaging there is no need to cover the surface, the thermal sensitivity is improved by a factor of 100, and there are less halos around local heat sources. The optical resolution of IR lock-in thermography, however, is limited by the IR wavelength of the order of $5 \mu \mathrm{m}$. There are also other applications of lock-in thermography like the imaging of the local distribution of gate oxide integrity (GOI) defects in large area MOS devices [11].

\section{Acknowledgments}

The authors are grateful to F. Altmann from FhG-IWM Halle and Micronas GmbH for their cooperation in the investigation of ICs. This work has been supported by the German BMBF under contract No. 0329743B

\section{References:}

[1] W. Gross, H. Scheuerpflug, Th. Hierl, M. Schulz and F. Karg, Proc. 2nd World Conference on Photovoltaic Energy Conversion, Wien (1998) 522.

[2] G. Aszodi, J. Szabon, I. Janossy und V. Szekely, Solid-State Electronics 24, (1981)1127 .

[3] P. Kolodner and J.A. Tyson, Appl. Phys. Lett. 40, (1982) 782 .

[4] X. Maldaque, Theory and Practice of Infrared Technology for Nondestructive Testing, WILEY, New York (2001).

[5] O.Breitenstein and M. Langenkamp, Sensors and Actuators A 71, (1998) 46.

[6] O.Breitenstein, M. Langenkamp, F. Altmann, D. Katzer, A. Lindner and H. Eggers, Rev. Sci. Instr. 71, (2000) 4155 .

[7] www.thermosensorik.com

[8] O.Breitenstein, M. Langenkamp, O. Lang, and A. Schirrmacher, Solar Energy Materials \& Solar Cells 65, (2001) 55 .

[9] M. Langenkamp, O. Breitenstein, M.E. Nell, A. Braun, H.-G. Wagemann, and L. Elstner, Proc. 16th Eur. Photovoltaic Solar Energy Conference, Glasgow 05/2000, 1644.

[10]H.S. Carslaw and J.C. Jaeger, Conduction of Heat in Solids, Oxford Clarendon (1959).

[11] S. Huth, O. Breitenstein, A. Huber and U. Lambert, J. Appl. Phys., 88, (2000) 4000 